\title{
AN EXPERIMENTAL MODEL OF PULMONARY CONTUSION FROM BLUNT THORCIC TRAUMA
}

\author{
Iv. Novakov ${ }^{1 *}$, Sv. Bachurska ${ }^{2}$, D. Delev ${ }^{3}$, D. Staikov ${ }^{2}$ \\ ${ }^{1}$ Department of Special Surgery, Medical University, Plovdiv, Bulgaria \\ ${ }^{2}$ Department of Pathology, Medical University, Plovdiv, Bulgaria \\ ${ }^{3}$ Department of Pharmacology, Medical University, Plovdiv, Bulgaria
}

\begin{abstract}
PURPOSE. The aim of this study was to present our own experimental model of pulmonary contusion from blunt thoracic trauma in small laboratory animals, accomplished by originally design platform.

METHODS. Fifteen Wistar albino rats (body weight 290, 0 - 320, 0) were used as the experimental object. Originally designed platform was used to cause blunt thoracic trauma in rats. Gross examination and histological evaluation of the contused lung were performed.

RESULTS. With the dosage of energy of the mechanical impact, achieved by our platform, pulmonary contusion was successfully provoked. Two of the most common mechanisms by which blunt thoracic trauma causes pulmonary contusion - compression of the chest wall against the lung parenchyma and "counter - coup" were reproduced. The experimental model that we accomplished managed to achieve bilateral pulmonary contusion by left - sided thoracic blow. Bilateral hemorrhages on the lateral surfaces of the lungs proven by gross and histological evaluation are evidence of pulmonary contusion. Histological analysis of the achieved samples demonstrates the considerable inflammatory cell-reaction in the contused lung areas by massive accumulation of neutrophil leucocites and macrophages $24^{\text {th }}$ hour after blunt thoracic trauma.

CONCLUSIONS. Our results demonstrated that with the originally designed platform we successfully created a reproducible experimental model of pulmonary contusion from blunt thoracic trauma in small laboratory animals. On the base of such studies some intimate mechanism of human pulmonary contusion can be explored.
\end{abstract}

Key words: rats, pulmonary contusion, blunt thoracic trauma, posttraumatic inflammatory response

\section{INTRODUCTION}

The pulmonary contusion still remains a significant problem in thoracic clinical practice. The pulmonary contusion is the most common intrathoracic injury, identified in blunt chest trauma (1, 2). Despite the development of intensive respiratory therapy, the mortality of patients with pulmonary contusion still remains in a very high level - $10 \%-25 \%$. The high mortality of pulmonary contusion explains the great interest in its experimental exploration (1, $2,3,4)$.

\footnotetext{
*Correspondence to: Ivan Petkov Novakov, Department of Special Surgery, Medical University, Plovdiv, "Peshtersko shosse" str. 66, Plovdiv 4004, Mob.phone: +359887 575487, e-mail: inovakov2003@yahoo.com
}

The aim of this study was to present our own experimental model of pulmonary contusion from blunt thoracic trauma in small laboratory animals, accomplished by originally design platform.

\section{MATERIAL AND METODS}

Fifteen male, Wistar albino rats (body weight 290,0 - 320, 0) were used as the experimental objects. Special platform, designed by ourselves, was used for this experimental study (Figure 1). The construction of the platform consists of several elements: solid wooden base; vertical metal rod, fixed to the base of the platform; horizontal metal rod, mobile towards vertical rod, outer plastic cylinder, fixed to horizontal rod; trigger, fixed to inner plastic cylinder; falling body (Figure 1). 
The falling body of the platform consists of plastic cylinder; base of metal plate with round shape; metal bolt that close the upper pole of the plastic cylinder. The plastic cylinder of the falling body is filled with pellets (diameter 0,75 $\mathrm{mm} /$ caliber 13 each). The small pellets that fill the plastic cylinder give the opportunity to change the weight of the falling body from 250 gr. to 950 gr. Four metal plates of the falling body with different diameters $(40 \mathrm{~mm}, 50 \mathrm{~mm}$, $60 \mathrm{~mm}$ and $70 \mathrm{~mm}$ ) were designed. By metal bolt that closes the upper pole of the plastic cylinder, the falling body is hanging on the trigger of the platform (Figure 1). By pressing the trigger the falling body is released from the horizontal rod and falls free onto the base of the platform. The construction of the platform makes it possible to change the height of the horizontal rod. It leads to changing the distance between falling body and the base of the platform. The animals were placed on their right site on the base of the platform, just under the falling body hanging on the trigger.

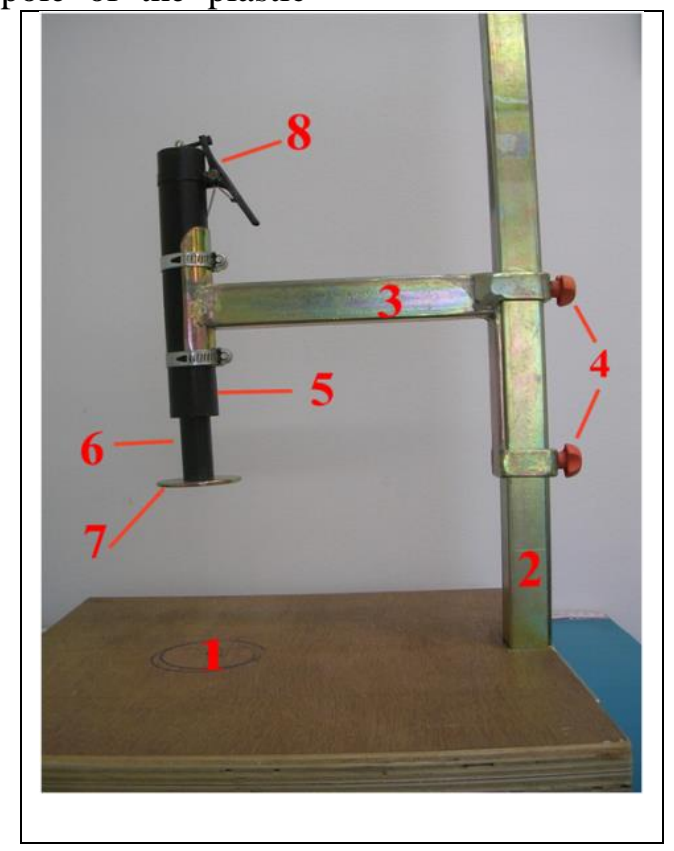

Figure 1. Platform for blunt thoracic trauma for small laboratory animals. (1 - wooden base; 2 - vertical metal rod; 3 - horizontal metal rod; 4 - metal bolts, which fixed horizontal metal rod; 5 - outer plastic cylinder; 6 - plastic cylinder of falling body; 7 - metal plate; 8 - trigger).

Blunt thoracic trauma was caused by free fall of the falling body upon the left thoracic wall of the animals. Kinetic energy of the mechanical impact on the thoracic cage of the animals is a function of the weight of the falling body and its height of dropping. The energy of the trauma (in joules) is calculated using the formula $\mathbf{E}=\mathbf{m} . \mathbf{g . h}$ (J) [m - mass of the falling body (kilograms); $g$ - gravitational acceleration $=9,81 \mathrm{~m} / \mathrm{s}^{2} ; \mathrm{h}-$ height that falling body is dropped from (meters)].

The animals were divided into five sub groups (by three in each) on the base of the kinetic energy of the impact on the thoracic wall: $1,8 \mathrm{~J}$, 2,0J, 2,2J, 2,4J и 2,6J. 500 gr. falling body was used. In order to generate mechanical impact with energies mentioned above 500 gr. should dropped down towards the thoracic cage of the animals from 37 sm., 41 sm., 45 sm., 49 sm., and $53 \mathrm{sm}$. respectively.

The animals were in state of general anesthesia (by intraperitoneal injection of ketamine hydrochloride $90 \mathrm{mg} / \mathrm{kg}$ ) at the moment of blunt thoracic trauma inducement. The animals were euthanized at the $24^{\text {th }}$ hour after the thoracic trauma by lethal dose of thiopental natrium (> $100 \mathrm{mg} / \mathrm{kg}$ ). The animals were immediately autopsied by opening the chest. The lungs were excised from the chest. Gross examination and histopatholocical evaluation were performed by two experienced laboratory pathologists independently.

The severity of pulmonary contusion was assessed by gross evaluation on the base of the hemorrhages on the lung surface. We had used the scoring system to determine the severity of 
the pulmonary contusion as followed: 1 hemorrhages up to $1 / 3$ of the surface of lung lobe; 2 - hemorrhages up to $2 / 3$ of the surface of lung lobe; 3 - hemorrhages more than $2 / 3$ of the surface of lung lobe. The severity of pulmonary contusion, on the base of our scoring system may varied from 1 to 18 and was determined as mild (score 1 to 2 ), moderate (3); severe (4 to 9) and very severe (10 and more).

Histological sections (stained with hematoxylin and eosin - H\&E) were examined by light microscopy under x 10, x 20, x 40 magnifications. The severity of histological lung changes, caused by blunt thoracic trauma was determined by scoring system as followed: $0-$ no changes, 1 -mild; 2 - moderate; 3 - severe.

The data of quantitative analysis of the severity of pulmonary contusion were expressed as mean with standard deviation (SD). The correlation between the energy of the blunt thoracic trauma and expression of the lung pathological changes was made by using the Spearman's coefficient.
This experimental study was conducted with the permission of the local ethics committee of Medical University, Plovdiv (№2/2012) and national food agency in Ministry of agriculture and food (№79/2013).

\section{RESULTS}

With the dosage of energy of the mechanical impact, achieved by our platform all fifteen rats had survived to the $24^{\text {th }}$ hour after accomplishing our experimental model. Pulmonary contusion was successfully provoked.

Gross pathology analysis established that leftsided lateral hit on the thoracic cage of the animals had caused pulmonary contusion that affected both lungs - left and right. Hemorrhages on both lateral surfaces of the lungs were established (Figure 2). On the posterior surface of the lung, adjacent to vertebral column and posterior segments of the ribs (costo-vertebral sulcus) the same findings were established/ (Figure 3).

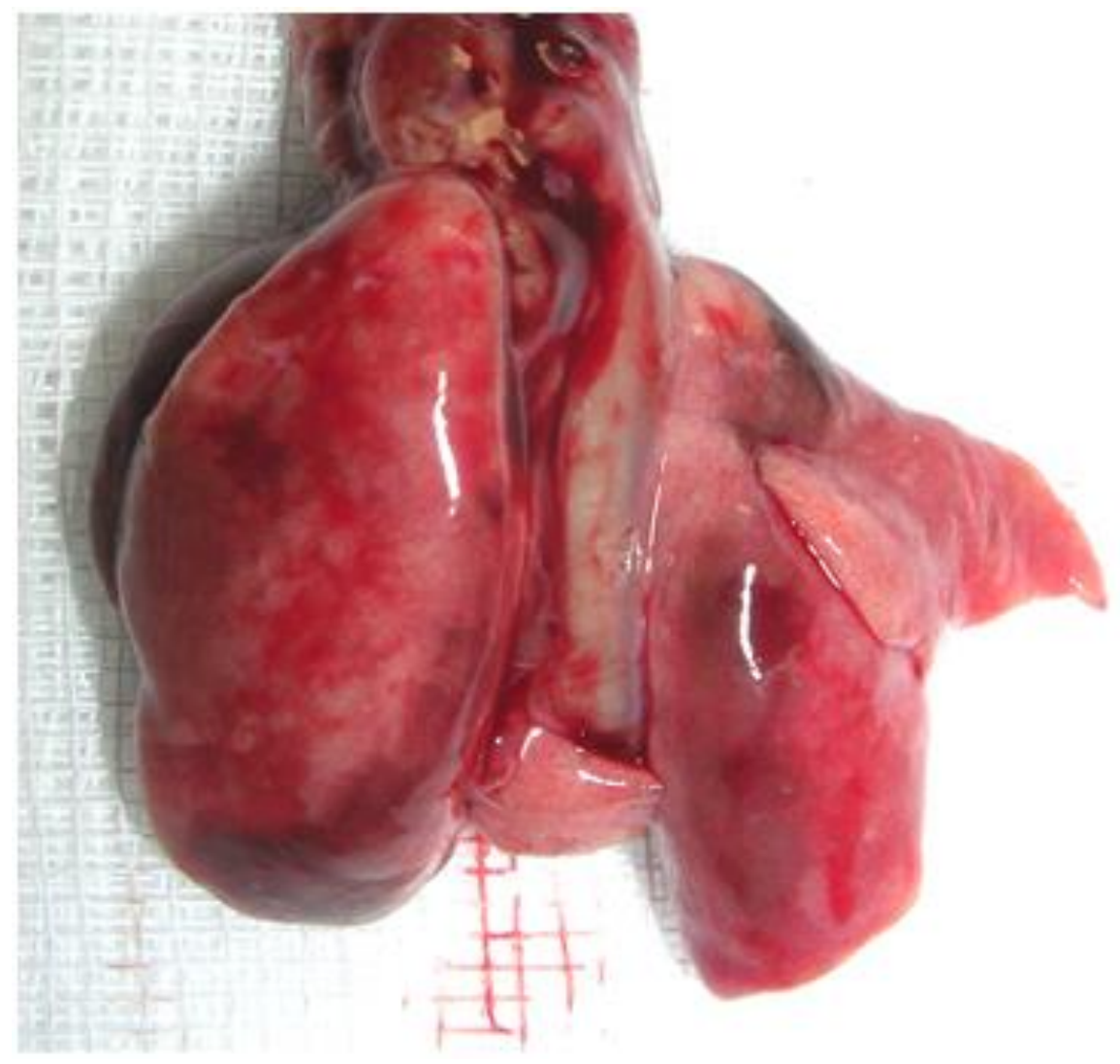

Figure 2. Pulmonary contusion - gross appearance. Hemorrhages on lateral surfaces of the both lungs. 


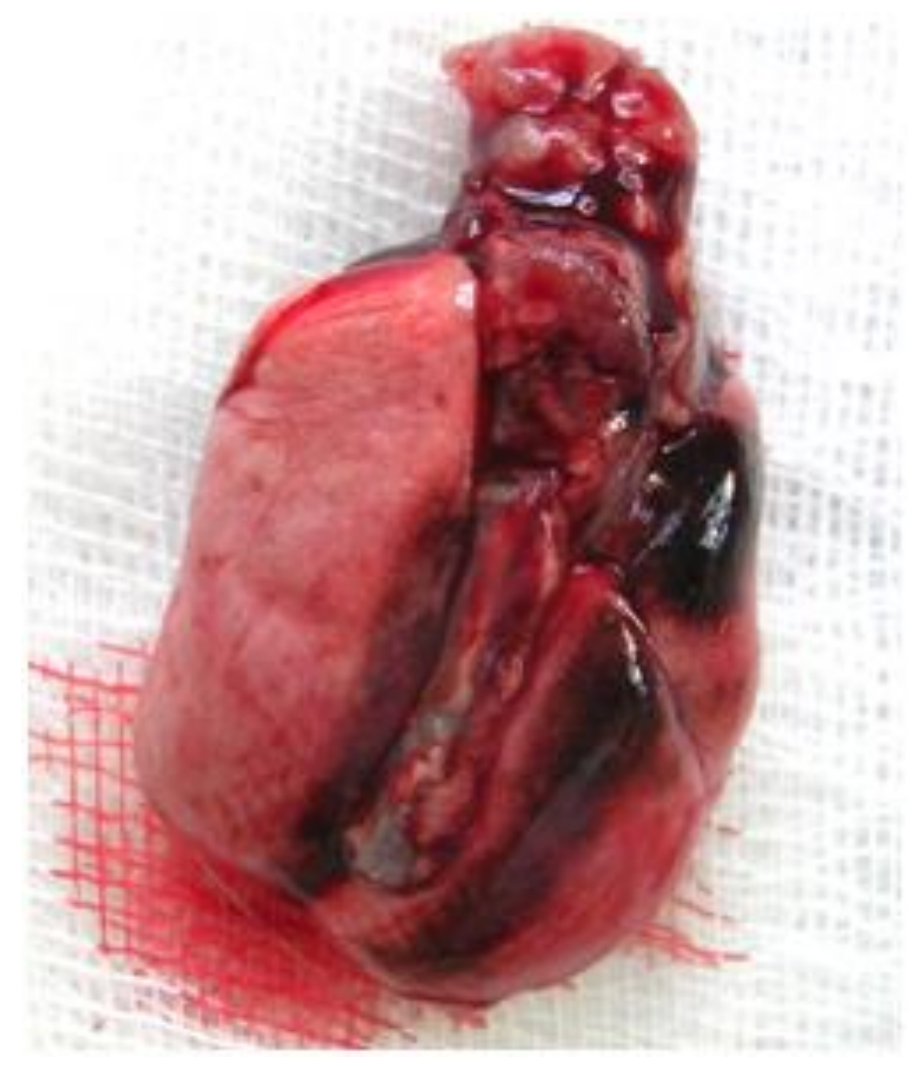

Figure 3. Pulmonary contusion - gross appearance. Hemorrhages on posterior surfaces of the both lungs.

Significant correlation between kinetic energy of mechanical impact on the chest wall and severity of the hemorrhages on the lung surface was found $(\mathrm{p}<0,001 /$ Spearman 'coefficient $(\mathrm{r})=1)$. Summarized results of gross pathology quantitative analysis are given in Table 1.

Table 1. Severity of the pulmonary contusion on the base of the kinetic energy of the blunt thoracic trauma - gross pathology analysis.

\begin{tabular}{|c|c|c|}
\hline energy of the impact $(\mathbf{J})$ & Score & severity of pulmonary contusion \\
\hline 1,8 & 1,0 & Mild \\
\hline 2,0 & 2,33 & mild; moderate \\
\hline 2,2 & 4,66 & Severe \\
\hline 2,4 & 5,33 & Severe \\
\hline 2,6 & 5,66 & Severe \\
\hline
\end{tabular}

Histological examination of the areas of pulmonary contusion found: parenchymal hemorrhages - interstitial and alveolar (Figure 4); alveolar destruction (Figure 5); injured bronchioles - with hemorrhages in their wall and lumen (Figure 6). Significant correlation between kinetic energy of mechanical impact on the chest wall and severity of the lung histopathological changes was established $(p<0,001 /$ Spearman's coefficient $(r)=1)$. Data of the quantitative analysis of lung pathological changes are given in Table 2. 


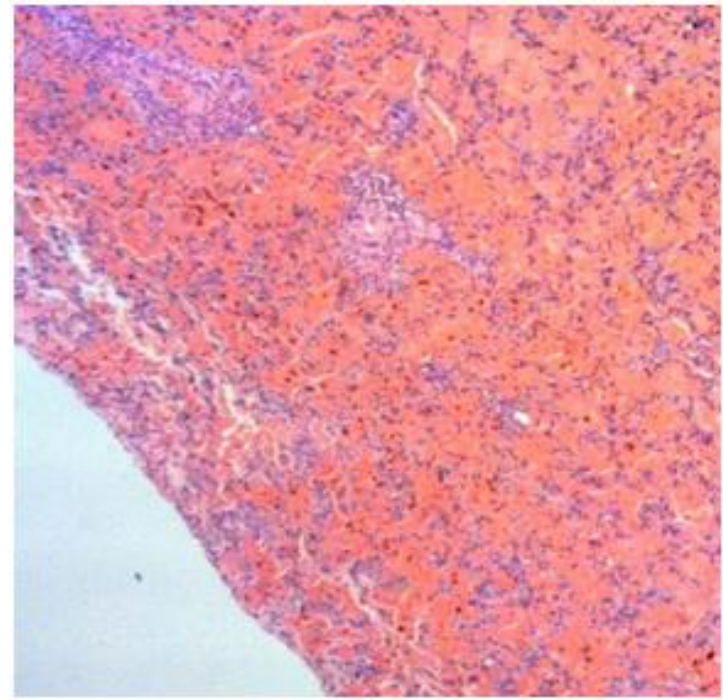

Figure 4. Pulmonary contusion - histological examination (H\&Ex10). Massive parenchymal (alveolar and interstitial) hemorrhage.

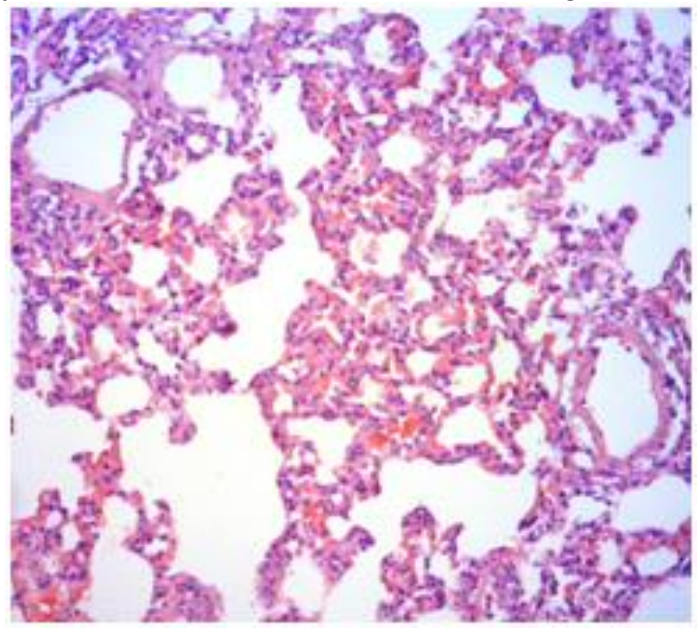

Figure 5. Pulmonary contusion - histological examination (H\&Ex20). Alveolar destruction is demonstrated.

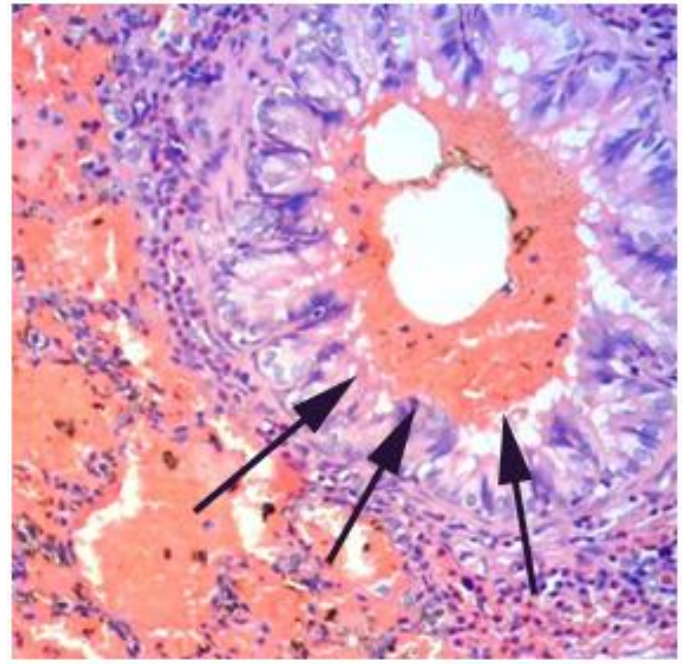

Figure 6. Pulmonary contusion - histological examination (H\&Ex20). Black arrows point damaged bronchioles, with hemorrhage in its wall and lumen. 
NOVAKOVIV, et al.

Table 2. Severity of the lung pathological changes in blunt thoracic trauma, on the base of the kinetic energy of the trauma - histological quantitative analysis.

\begin{tabular}{|l|l|l|l|l|l|}
\hline \multirow{2}{*}{ Lung pathological changes } & \multicolumn{5}{|c|}{ Energy of the impact } \\
\cline { 2 - 6 } & $1,8 \mathrm{~J}$ & $2,0 \mathrm{~J}$ & $2,2 \mathrm{~J}$ & $2,4 \mathrm{~J}$ & $2,6 \mathrm{~J}$ \\
\hline Parenchymal hemorrhage & 1,00 & 1,33 & 1,83 & 2,50 & 2,83 \\
& $(0)$ & $(0,52)$ & $(0,41)$ & $(0,55)$ & $(0,41)$ \\
\hline Alveolar destruction & 1,00 & 1,50 & 2,16 & 2,50 & 3,00 \\
& $(0)$ & $(0,54)$ & $(0,41)$ & $(0,54)$ & $(0)$ \\
\hline \multirow{2}{*}{ Bronchiolar injuries } & 0,83 & 1,16 & 1,50 & 2,16 & 2,83 \\
& $(0,41)$ & $(0,41)$ & $(0,54)$ & $(0,41)$ & $(0,41)$ \\
\hline
\end{tabular}

Histological examination had also established acute inflammatory-cell response of the lung contusion areas (Figure 7). There is massive accumulation of polymorphonuclear cells (neutrophil leucocytes) and moderate infiltration of mononuclear cells (macrophages). Granules of hemosiderin were established in the cytoplasm of many of macrophages (hemosiderophages) / (Figure 8).

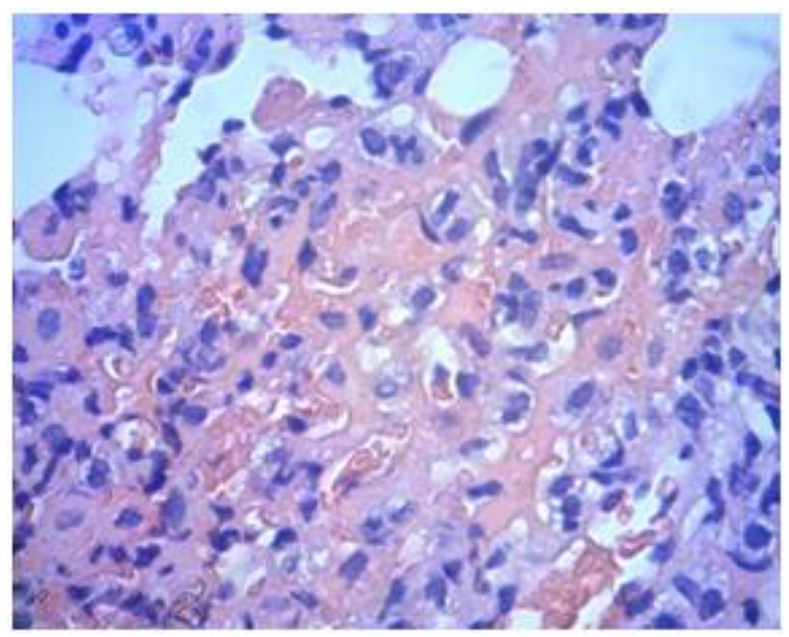

Figure 7. Posttraumatic acute inflammatory-cell response of contused lung at $24^{\text {th }}$ hour after blunt thoracic trauma - histological examination [H\&Ex20].

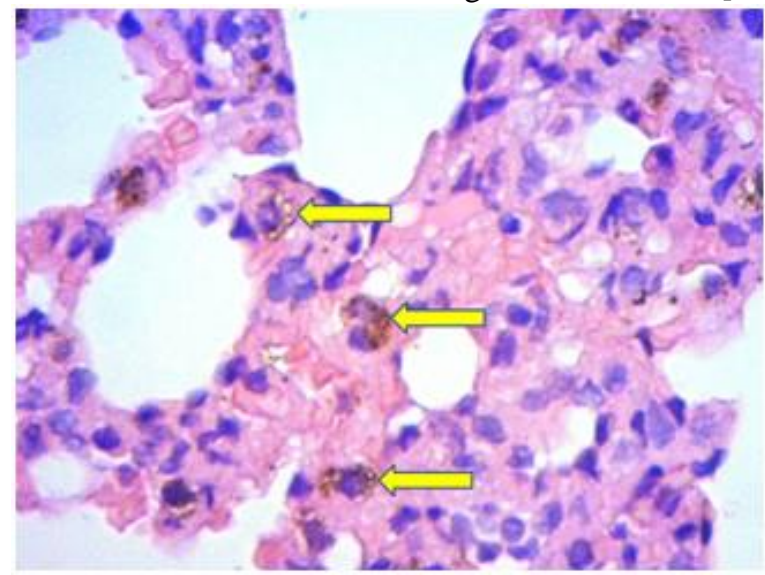

Figure 8. Contused lung at $24^{\text {th }}$ hour after blunt thoracic trauma - histological examination. Yellow arrows point hemosiderophages - [H\&Ex40]. 


\section{DISCUSSION}

Because of its high incidence and high level of mortality, the pulmonary contusion is considered as a serious problem in thoracic surgery practice. These characteristics of pulmonary contusion explain the great interest in its experimental exploration $(1,2,3)$. Our scientific work and interest needed the creation of experimental model of pulmonary contusion, which is presented in this study.

The first task to solve was to choose the species of animals for our model of pulmonary contusion. There are limitations in using the large-animal models of pulmonary contusion today. Some reasons for such limitations are: complex technical supply, frequent mortality of the animals and high cost of the experiments (1, $5,6,7)$. Because of that, many authors have used small laboratory animals for their experimental models of pulmonary contusion $(1,8,9,10)$.

We took the decision to use Wistar albino rats for our experimental model of pulmonary contusion. Wistar albino rats have great fertility, high resistance to different unfavourable impacts (like anesthetics and mechanical impact). There are also many cell- and mediator - specific reagents, which are widely available for small animals such as rats $(1,2)$. These facts can explain our choice of laboratory animal for our experimental model of pulmonary contusion.

Nowadays experimental pulmonary contusion most commonly is caused by mechanical impact by falling solid body onto the chest wall of the laboratory animals $(8,9,10)$. This mechanism of lung damage was accepted by our scientific team. We had designed and constructed a special platform, unique for this study. The characteristics of this platform are: a simple construction, easily to use, precisely determination of the kinetic energy of mechanical impact on the thoracic cage of the animals.

Expectantly for us, a significant correlation between kinetic energy of mechanical impact on the chest wall of the animals and severity of pulmonary contusion changes (macroscopic and histological) was established. Our results confirmed that even with relatively high energy of mechanical impact $(2,4 \mathrm{~J} ; 2,6 \mathrm{~J})$ the animals would survive after blunt thoracic trauma $(8,9$, 10).
The experimental model that we accomplished managed to achieve bilateral pulmonary contusion by left - sided thoracic blow. Bilateral hemorrhages on the lateral surfaces of the lungs proven by gross and histological evaluation are evidence of pulmonary contusion. The presence of hemosiderophages was demonstrated. Hemosiderin is produced by macrophages after 18 to 20 hours in areas of tissue hemorrhages, which are caused by blunt trauma. That's why hemosiderophages, which were established on histological sections, are evidence that pathological changes of the lung are caused by blunt thoracic trauma (not by iatrogenic lung damage trough the autopsy of the animals).

Two simultaneously acting pathophisiological mechanisms - compression of the chest wall against the lung parenchyma and "counter coup" are the reason for such changes. Mechanical impact on the left thoracic wall causes left-sided pulmonary contusion. At the moment of left-sided blow, the right lung is also under compression - the result of compression of the right chest wall toward the solid base of the platform. It explains why left-sided lateral mechanical impact on the thoracic cage causes bilateral pulmonary contusion. Sudden compression of the chest wall on the lung at the moment of blunt thoracic trauma causes sudden movement of the lung against the rigid vertebral column and posterior segments of the ribs. The compression of the lung against rigid vertebral column causes pulmonary contusion by "counter - coup" mechanism (11). We had demonstrated hemorrhages on the posterior surface of the lungs, the result of counter - coup mechanism of pulmonary contusion. The platform we used and the model we accomplished reproduced two of the most common mechanisms of human pulmonary contusion in real life.

Clinical and experimental studies had established that pulmonary contusion is a trigger for the development of posttraumatic acute inflammatory response of the lung $(1,2,3,7,12$, 13). This inflammatory process may progress to severe lung edema, with extremely high risk of death of the patients $(7,12,13)$. Experimental studies had established that at the $24^{\text {th }}$ hour after the blunt thoracic trauma there is considerable inflammatory cell-reaction in the contused lung areas $(6,7,9,10)$. Because of posttraumatic inflammatory cell reaction, the pulmonary architecture is completely obliterated in affected 
areas of the lung $(6,7)$. Histological analysis of the achieved samples demonstratively confirmed such progression of pulmonary contusion by massive accumulation of neutrophil leucocytes and macrophages. These finds support the usefulness of the platform we used and the experimental model we desired in representing a real model of pulmonary contusion comparable to other published models.

\section{CONCLUSIONS}

We have presented a reproducible small-animal experimental model of pulmonary contusion from blunt thoracic trauma. This model gives us the opportunities for further study of different aspects of pulmonary contusion and progression of posttraumatic lung pathological changes. Our results demonstrated that with the originally designed platform we successfully created a reproducible experimental model of pulmonary contusion from blunt thoracic trauma in small laboratory animals. Furthermore, construction of the platform gives us the opportunity to cause, reproduce and explore experimental blunt trauma in other anatomical body regions (not only thoracic trauma). The observations of such studies can explore some intimate mechanism of human pulmonary contusion.

\section{REFERENCES}

1. Cohn SM, DuBose JP. Pulmonary contusion: an Update on Recent Advances in Clinical Management. World J Surg 2010; 34:1959-1970.

2. Cohn SM. Pulmonary contusion: review of the clinical entity. J Trauma; 42(5): 973979, 1997.

3. Allen GS, Coates NE. Pulmonary contusion: a collective review. Am Surg; 62: 656-669, 1996.
4. Wanek S. Blunt thoracic trauma: flail chest, pulmonary contusion and blast injury. Crit Care Clin; 20: 71-81, 2004.

5. Hellinger A, Konerding M, Malkusch W et al. Does lung contusion affect both the traumatized and the noninjured lung parenchyma? A morphological and morphometric study in the pig. J Trauma; 39(4): 712-719, 1995.

6. Moseley R, Major M, Jerome $\mathrm{J}$ et al. Response to blunt chest injury: a new experimental model. J Trauma; 10(8): 673683, 1970

7. Fulton RL, Peter ET. The progressive nature of pulmonary contusion. Surgery;67:499506, 1970.

8. Özel SK, Özel HB, Colakoglu NM et al. Protective effect of the thoracic cage on parenchyma in response to trauma in blunt thoracic trauma: an experimental study. Turkish Journal of Trauma; 16 (4):287-292, 2010.

9. Raghavendran K, Davidson BA, Helinski $\mathrm{EH}$ et al. A rat model for isolated bilateral lung contusion from blunt chest trauma. Anesth Analg; 101:1482-1489, 2005.

10. Wang ND, Stevens MH, Doty DB et al. Blunt chest trauma: an experimental model for heart and lung contusion. J Trauma; 54:744-748, 2003.

11. Wagner RB, Crawford WO, Schimpf PP. Classification of parenchymal injuries of the lung. Radiology; 167:77-83, 1988.

12. Hoff SJ, Shotts SD, Eddy VA, et al. Outcome of isolated pulmonary contusion in blunt trauma patients. Am Surg;60:138142, 1994.

13. Johnson JA, Cogbill TH, Winga ER. Determinants of outcome after pulmonary contusion. J Trauma;26:695-697, 1986. 